\title{
The Influence of Stressful Environmental Stimuli on Changes of Catecholamines and Blood Pressure
}

\author{
Nagao Kajtwara, M.D., Yoshiko Kobayashi, M.D., Akira \\ Murakami, M.D., Jun Hashida, M.D., Takahisa \\ UchiYama, M.D., Takashi KITA, M.D., Masahiko \\ Sato, M.D., Yoshihide Okumura, M.D., \\ Masashi Ono, M.D., Takeshi MrkamI, M.D., \\ and Kenzo Oshima, M.D.
}

The effects of stressful environmental stimuli on the development of hypertension have been studied in spontaneously hypertensive rats (SHR). We have applied several kinds of stresses to SHR. They were divided into stress and nonstress groups.

1) Noisy enviornment: SHR of the stress group were housed in the noisy mixed animal house from 4 weeks after birth for 22 weeks. Nonstress group was housed in the quiet same animal house. During stress-loading SHR showed increases in their blood pressure, adrenal weight and catecholamines in adrenals and brain, while norepinephrine in heart was decreased.

2) Acute cold exposure: SHR of the stress group were housed in the same animal house kept in a cold environment from 10 months after birth for 4 weeks. Nonstress group was kept under the warm conditions of temperature. Blood pressure and catecholamines in adrenals, brain and blood in the exposed were higher than those in the unexposed, while norepinephrine in heart was lower.

3) Combined visual and auditory stimuli: The stress group was put in "stress" cage and exposed to combined stress of a high-pitched buzzer (duration 5 sec 6 times per min) and flickering of incondescent lamps of $100 \mathrm{v}$, $100 \mathrm{w}$ in the cage ( 60 times per $\mathrm{min}$ ) for 2 hours daily from 13 months after birth for 4 weeks. Blood pressure in the stress group was higher in the mean than in the nonstress group. Catecholamines in adrenals and brain were not different between them, while blood norepinephrine in the former was higher and heart norepinephrine was lower than the latter.

From the Department of Cardiovasology, Surugadai Nihon University Hospital, Tokyo. 